\title{
Performance Evaluation of Energy-Efficient Game- Theoretic Resource Allocation Schemes in OFDMA Cellular Networks
}

\author{
Emmanuel Duarte-Reynoso, Domingo Lara-Rodríguez \\ Electrical Engineering Department, CIVESTAV-IPN, México \\ \{eduarte, dlara\} @einvestav.mx
}

\begin{abstract}
In this paper, we study the performance of energy-efficient resource allocation schemes for multi-cell OFDMA networks based on Game Theory. These schemes have been evaluated in the literature; however, the evaluations have been done under different system models and simulation environments, making a fair comparison unfeasible. Hence, we first develop an appropriate system model for comparison. Then, we analyze the implementation of the selected schemes under constraints introduced by the proposed system model. Finally, throughout extensive numerical simulations on a realistic environment, we evaluate the performance and tradeoffs between energy efficiency, throughput, outage probability and fairness, highlighting the need for some scheme that improves the outage probability and the throughput maintaining high energy efficiency.
\end{abstract}

Keywords: resource allocation, OFDMA, energy efficiency, game theory.

\section{Introduction}

The growing demands of higher speed and reliable communications in nextgeneration wireless networks have motivated to adopt OFDMA in the current proposals for this kind of networks (3GPP LTE and IEEE $802.16 \mathrm{~m}$ for instance) due to its robustness and flexibility. Dynamic resource allocation allows OFDMA systems to exploit multi-user diversity leading to a significant performance improve in terms of throughput, fairness and efficiency. Due to this feature, the problem of resource allocation in OFDMA has caught researchers' attention in the last years (e.g. [1-3]).

Resource allocation schemes are designed with a specific objective, typically capacity maximization [4]. However, the need to transmit user generated content and the limited power available at user devices has motivated researchers to address the resource allocation problem in OFDMA through an energy-efficient approach. Nevertheless, the subcarrier assignment problem as demonstrated in [5] is a non-convex optimization problem thus, the global optimization of this problem is unfeasible and some alternative methods should be studied to address it.

Besides, game theory [6] that focuses in distributed solutions to agent interaction problems is presented as a valuable tool that enables the abstraction of resource allo- 
cation problem through a different approach and the application of its fundamental results to simplify the analysis of this problem. Numerous energy-efficient gametheoretic allocation schemes have been proposed in the open literature (e.g. [8-14]). Our aim in this paper is to establish a common system model to perform a comparison between some selected of these schemes.

This paper is structured as follows. Next section describes the system model used in the comparison. Section 3 gives an overview of the compared schemes. Section 4 presents the simulation environment as well as the corresponding numerical results. Last, conclusions are presented in Section 5.

\section{System Model}

The system model presented is based on the 3GPP LTE physical layer specifications [15]. We consider the uplink channel of a multi-cell OFDMA network with universal frequency reuse. Let $M=\{m, m=1,2, \ldots|M|\}$ denote the set of cells in the network, and $K=\left\{k, k=1,2, \ldots,\left|K_{m}\right| \cdot|M|\right\}$, the set of active users (mobile stations) with $K_{m}=\left\{k \mid m_{k}=m\right\}$ the set of active users in cell $m$, where $m_{k}$ is the base station to which user $k$ is assigned.

The available spectrum is divided into physical resource blocks (PRBs); each PRB is formed by $N_{b}$ subcarriers with frequency spacing $B W$, so we let $N$ be the set of PRBs in the system. Each cell $m$ allocates the complete set of PRBs among its active users through the allocation matrix $A=\left\{a_{m, n}\right\}$, where element $a_{m, n}$ indicates the user to which PRB $n$ has been allocated. PRB allocation is repeated each transmission interval which consists of $D_{s}$ signaling symbols and $D_{d}$ user information symbols.

It is assumed that the receiver and the transmitters have perfect channel state information (CSI) and that it is possible to use a single coefficient $h_{m, k}^{2}(n)$ to represent the channel power gain between user $k$ and base $m$ in PRB $n$. The transmit power vector of user $k$ is denoted by $p_{k}=\left\{p_{k, n} \mid p_{k, n} \geq 0\right\}$, where element $p_{k, n}$ indicates the transmit power of the user for every subcarrier in PRB $n$. This vector has the following restrictions:

$$
\begin{gathered}
p_{k, n}=0, a_{m_{k}, n} \neq k \\
\sum_{n \in N} p_{k, n} \leq \frac{P_{\max }}{N_{b}}
\end{gathered}
$$

where (1) prevents the existence of intra-cell interference and (2) stands for the limited power source at mobile station. 
We consider the effect of additive white Gaussian noise (AWGN) with power $\sigma^{2}$ at the receiver; thus, the signal to noise plus interference ratio (SINR) of user $k$ at base $m$ such that $a_{m, n}=k$ over PRB $n$ is computed as:

$$
\gamma_{m, n}=\frac{p_{k, n} h_{m, k}^{2}(n)}{\sigma^{2}+I_{m, n}}
$$

where $I_{m, n}$ is the power of the interference received at base $m$ over PRB $n$ :

$$
I_{m, n}=\sum_{j \in K \mid j \neq k} p_{j, n} \cdot h_{m, j}^{2}(n) .
$$

Given the SINR $\gamma_{m, n}$ the goodput at base $m$ over PRB $n$ is obtained as:

$$
r_{m, n}=\frac{D_{s}}{D_{s}+D_{d}} N_{b} \cdot B W \cdot e\left(\gamma_{m, n}\right)
$$

where $e\left(\gamma_{m, n}\right)$ is an efficiency function dependent of the modulation used by the mobile station, that gives the effective data rate in bps/Hz. Energy efficiency is computed for user $k$ transmitting to base $m$ over PRB $n$ as

$$
u_{k, n}=\frac{r_{m, n}}{p_{k, n}}, a_{m, n}=k
$$

Finally, we define the no-transmission probability as the probability that in a given transmission interval, the total transmitted power of a user is equal to zero, i.e. $\sum_{n \in N} p_{k, n}=0$.

\section{Energy-Efficient Resource allocation schemes}

Since the seminal works presented by Goodman et al [16] many game theoretic energy-efficient power control and subcarrier allocation schemes have been reported in the literature (e.g. [8-14]). As stated before, we aim to compare the performance of these schemes; however, it is worthless to make a comparison between all of them because some schemes are not feasible or simply it has been demonstrated that they are outperformed by others. Hence, we have selected three schemes for comparison, taking into account that their system models are close to the one presented in section 2 and that they consider both, subcarrier and power allocation. In the following, we give a brief description of these schemes. 


\subsection{Scheme 1}

The first scheme is the one presented in [13]. In this scheme the problem is decoupled into subcarrier assignment and a subsequent power allocation. Subcarrier assignment is done in a centralized manner, i.e. it is performed by the base stations. To address this problem, the authors propose a heuristic algorithm to be executed by each base station in the network.

This algorithm divides the set of subcarriers $N$ into $C$ clusters denoted as $N^{(1)}, \ldots, N^{(C)}$, where each cluster has a number of subcarriers equal to the number of active users in the cell. In order to provide farther users with a significant performance and, in this way improve the fairness of the allocation, the algorithm tries to allocate one subcarrier per user in each cluster. This is done by allocating each user with its best available subcarrier starting by the users with the lowest channel gains. Nevertheless, this could induce negative effects to the global performance if users are experiencing deep fading conditions. To limit such negative effects, no subcarriers are allocated to users with too weak channel conditions. To place and adaptive threshold, it is prevented a user from receiving any subcarrier if its channel gain in its best subcarrier is $\lambda$ times lower than the highest one in that subcarrier. The value of the threshold is set in the interval $0<\lambda<1$ which allows to trade off performance and fairness in the network.

When subcarrier assignment has been done by the base stations, the power control is addressed as a non cooperative game between the users in the system considering QPSK fixed modulation. Particularly, based on the results on [16], a best-response dynamic is proposed in which user $k$ adopts a transmit power

$$
p_{k, n}^{*}=\min \left(p_{\max }, \frac{\gamma_{k}^{*}\left(\sigma^{2}+I_{m, n}\right)}{h_{m, k}^{2}(n)}\right)
$$

where $\gamma_{k}^{*}$ is the SINR such that

$$
\frac{f\left(\gamma_{i_{k}}^{*}\right)}{\gamma_{i_{k}}^{*}}=\left.f^{\prime}\left(\gamma_{i_{k}}\right)\right|_{\gamma_{i_{k}}=\gamma_{i_{k}}^{*}}
$$

where $f(\gamma)$ is the efficiency function for the used modulation, defined in (5) as $e(\gamma)$. The model considered in scheme 1 is very close to that proposed in section 2 . We only introduce the constraint of a minimum SINR threshold that has to be achieved and we consider the allocation of PRBs instead of subcarriers.

\subsection{Scheme 2}

Scheme 2, was first presented in [12]. Here, the authors claim that due to the difficulty of optimizing the non-convex function of the sum of energy efficiency, it is 
more feasible to maximize it in a time-averaged manner instead taking an averaging window of size $w$.

This scheme proposes a framework where the base stations are the players that form $|N|$ independent non-cooperative games, one game per subcarrier. In each game, at transmission time interval $t$, the base stations will attempt to maximize the average efficiency in the corresponding subcarrier, allocating it to the user with the greatest ratio between optimal achievable rate $r_{m, n}^{k^{*}}$ and its average transmit power at $t-1 \quad P_{k, n}(t-1)$. It is considered that users are capable of select its modulation scheme adaptively from a set $Q$ so that the optimal achievable rate could be not feasible; thus, it is necessary to define an efficiency gap:

$$
\Delta g_{m, n}^{k}(t)=\min _{q \in Q}\left|\tilde{r}_{q}-r_{m, n}^{k^{*}}(t)\right|
$$

where $\tilde{r}_{q}$ is the rate corresponding to modulation scheme $q \in Q$. Hence, each subcarrier is assigned to the user that maximize

$$
\eta_{m, n}^{k}(t)=\frac{\hat{r}_{m, n}^{k^{*}}(t)-\beta \Delta g_{m, n}^{k}(t)}{P_{k, n}(t-1)}
$$

where $\beta$ is a design parameter that takes values between 0 and 1 . After this, each user adopts the modulation scheme that minimizes its efficiency gap and the transmit power need to achieve the SINR threshold corresponding to this modulation scheme.

It is worth noting that in the proposed game, a change in the strategy of a base station might change the assigned user which usually results in different interfering power to other cells, so other cells will continue the best response dynamic until an equilibrium point is reached. However, the game doesn't guarantee to converge to an equilibrium so it is necessary to limit the maximum iteration time to prevent infinite loop.

Some refinements are needed to implement this scheme considering the model proposed in section 2. First, we consider the modulation schemes and its BLER curves reported in [17]; those curves give us the information needed to compute the efficiency function $e\left(\gamma_{m, n}\right)$ and the goodput in (5). Second, this scheme does not consider the case where a user is not capable to achieve the minimum SINR threshold, so we established that in this case, the user set its transmit power to cero. Finally, as we stated for scheme 1, we consider PRBs instead of subcarriers.

\subsection{Scheme 3}

This last scheme proposed by Buzzi et al [10], resorts to potential game theory as a potential game is guaranteed to converge to the Nash equilibrium point through the best (or better) response dynamic. It is considered a strategy space in which each user 
choose an equal number of subcarriers $L$ as well as its transmit power aiming to maximize the system energy efficiency.

Taking into account these considerations, a non-cooperative potential game is proposed. The potential function is defined as the natural logarithm of the efficiencies of each user over each subcarrier:

$$
V=\ln \left(\prod_{k=1}^{K} \prod_{\substack{n \in N, a_{m, n}=k}} \frac{e\left(\gamma_{m, n}\right)}{p_{k, n}}\right)
$$

where $e\left(\gamma_{m, n}\right)$, the efficiency function of the fixed QPSK modulation is defined as:

$$
e\left(\gamma_{m, n}\right)=\left(e^{-\beta / \gamma_{m, n}}\right)^{D_{d}}
$$

where $\beta=\gamma^{*} / M$, and $\gamma^{*}$ is the optimum SINR defined by (8).

In [10] it is shown that $V$ is the potential function when the user's utility is given by

$$
\begin{aligned}
u_{k}\left(a_{m_{k}, n}, p_{k, n}\right) & =-\beta D_{d} \sum_{\substack{n \in N \\
a_{m, n}=k}} \frac{\sigma^{2}+\sum_{j=1, j \neq k}^{K} p_{j, n} h_{m_{k}, j}^{2}(n)}{p_{k, n} h_{m_{k}, k}^{2}(n)} \\
& -\beta D_{d} \sum_{\substack{i=1, i \neq k \\
a_{n \in N}}} \sum_{\substack{a_{i, n}=i \\
\text { nan }}}\left(\frac{p_{k, n} h_{m_{i}, k}^{2}(n)}{p_{i, n} h_{m_{i}, i}^{2}(n)}\right)-\sum_{\substack{n \in N \\
a_{m, n}=k}} \ln \left(p_{k, n}\right)
\end{aligned}
$$

This indicates that the best response dynamic, in which every user updates its subcarriers and transmit power to maximize (13) (in a combinatorial fashion) will converge to a Nash equilibrium point. Once that we have presented this scheme some aspects have to be pointed out with respect to the model presented in section 2 .

As it was established, all the PRBs have to be allocated; thus, the value of $L$ becomes a function of the number of active users in each cell. The authors don't impose any constraint to prevent the frequency reuse within the cell, so the best dynamic response could lead to this situation. If so, we set the next rule: if more than one user is allocated a given subcarrier the base station takes a decision, allocating the subcarrier to the user which achieves the higher efficiency. Due to the need of the base station intervention and the form of the potential function, which require CSI from all users this scheme is considered as a centralized scheme. In order to reduce the computational complexity of this scheme, which comes from the combinatorial search performed for each user, we adopt the sub-optimal implementation proposed in [10]. As in the previous cases, we consider the allocation of PRBs instead of subcarriers and a minimum SINR threshold $S_{\min }$. 


\section{$4 \quad$ Numerical results}

\subsection{Simulation setup}

In order to compare the presented schemes under realistic conditions, we set our simulation environment according to [15]. We considered a 19 cell grid, i.e. a center cell and the first two tiers of surrounding cells. To mitigate the border effect we make use of the wrap-around technique [18]. Cell radius is $R=1000 \mathrm{~m}$.

The number of PRBs available in the system is $|N|=50$, these PRBs consist of $N_{b}=12$ subcarriers and the space between subcarrier frequencies is $B W=15 \mathrm{kHz}$. Location of active users follows a uniform distribution restricted to distances larger than $35 \mathrm{~m}$ from the base station. Maximum transmission power per user $P_{\max }$ is set to $21 \mathrm{dBm}$ and noise power spectral density at base stations is $N_{0}=-170 \mathrm{dBm} / \mathrm{Hz}$. Channel coefficients are computed as:

$$
h_{m, k}^{2}(n)=\mu_{m, k} \hat{h}_{m, k}^{2}(n)
$$

where $\hat{h}_{m, k}^{2}(n)$ models fast fading and is computed from ITU pedestrian-A channel profile [19]; while $\mu_{m, k}$ is a frequency-independent factor which includes shadowing and path-loss effects:

$$
\mu_{m, k}=10^{\frac{-L_{m, k}+X_{\sigma}}{10}}
$$

where $X_{\sigma}$ is a gaussian random variable with standard deviation of $10 \mathrm{~dB}$ and $L$ represents path-loss which is computed as:

$$
L_{m, k}=128.1+37.6 \log _{10}\left(\frac{d_{m, k}}{d_{0}}\right)
$$

where $d_{m, k}$ is the distance between user $k$ and base $m$; and $d_{0}$ is the reference distance equal to $1 \mathrm{~km}$. We consider a transmission time interval of $1 \mathrm{~ms}$, having $D_{s}=4$ signaling symbols and $D_{d}=10$ information symbols.

The parameters concerning each compared scheme are detailed in the following. According with the results reported in [13], we set the value of threshold $\lambda$ to $-20 \mathrm{~dB}$ for scheme 1 . The averaging window size of scheme 2 is $w=10$ and parameter $\beta$ was set to 0.7 . We consider a minimum SINR threshold for all schemes equal to -1.43 $\mathrm{dB}$. 


\subsection{Simulation results}

Having established a common simulation environment we are able to compare the performance of the resource allocation schemes. This subsection presents the results for different performance metrics as a function of the distance between the user and its base station considering a fixed number of users per cell $\left|K_{m}\right|=20$.

Fig. 1 shows the average efficiency performance. As can be seen scheme 1 achieves the largest efficiency for distances shorter than $250 \mathrm{~m}$ while scheme 2 outperforms it for larger distances. Efficiency decays with the distance as farther users require more power to achieve the same SINR that center users. Nevertheless it is worth noting that all three schemes decay with a different rate, in particular we observe that this rate is higher for scheme 1, followed by scheme 2 and by scheme 3 .

To have a better understanding of what happens with efficiency, we can resort to the results of Fig. 2 and Fig. 3 which present the average goodput and average transmit power respectively. Scheme 1's goodput is the greater for distances less than 750 $\mathrm{m}$. However its transmit power is not the lowest, hence it is outperformed in terms of efficiency by scheme 2 which achieves an intermediate goodput and maintains the lowest transmit power. This is done by means of the modulation adaptation considered in scheme 2. On the other hand, scheme 3 has the lowest goodput and the highest transmit power which results in a low efficiency, which is due to the PRB assignment rule in scheme 3 that tries to introduce some fairness by allocating the same number of PRBs per user. By doing so, the performance gap between cell-center users and edge users is reduced but the goodput for all users is reduced as well.

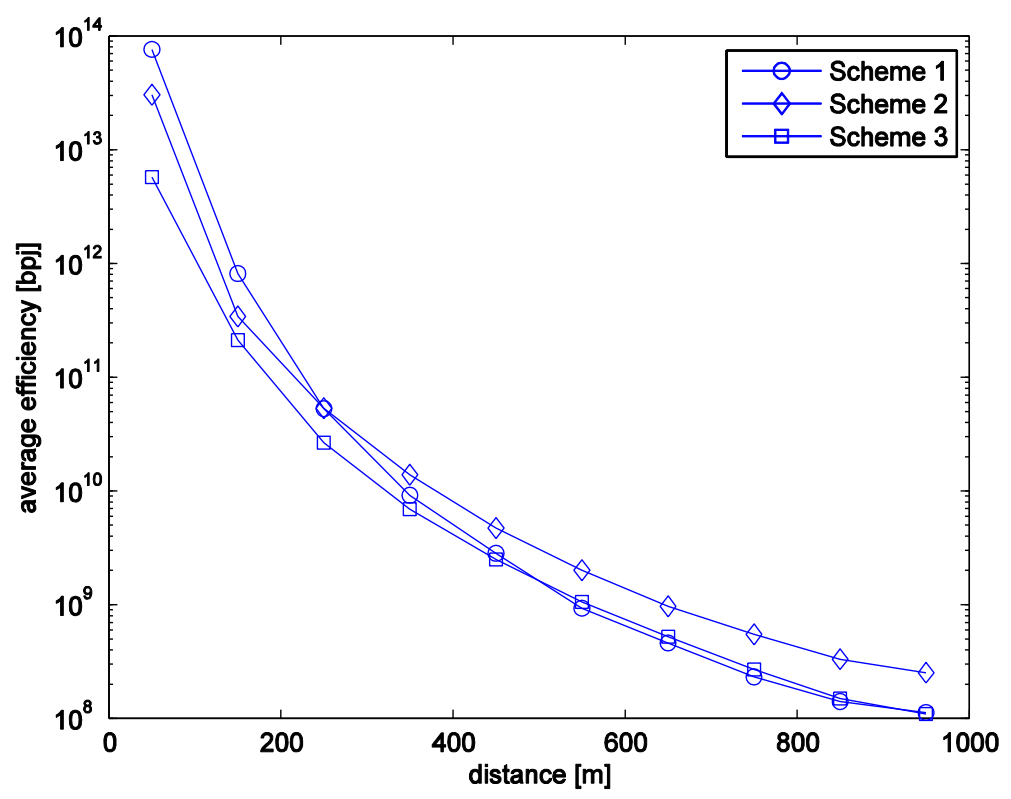

Fig. 1. Average energy efficiency as a function of the user distance 
Performance Evaluation of Energy-Efficient Game-Theoretic Resource Allocation Schemes ...

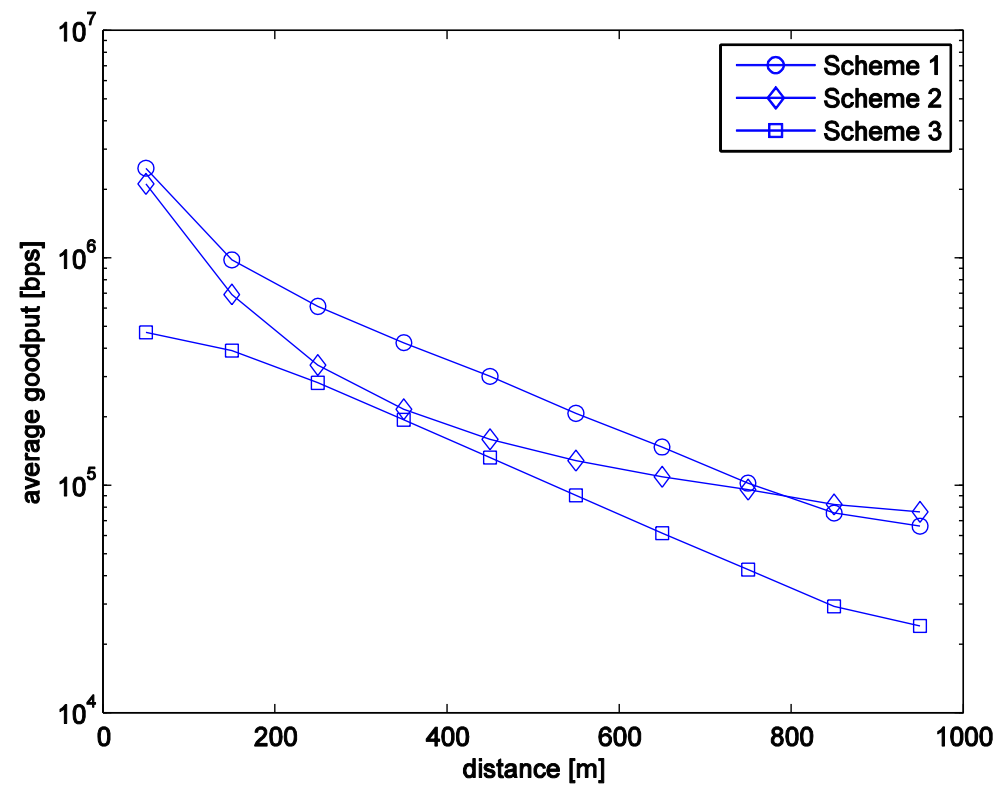

Fig. 2. Average goodput as a function of the user distance

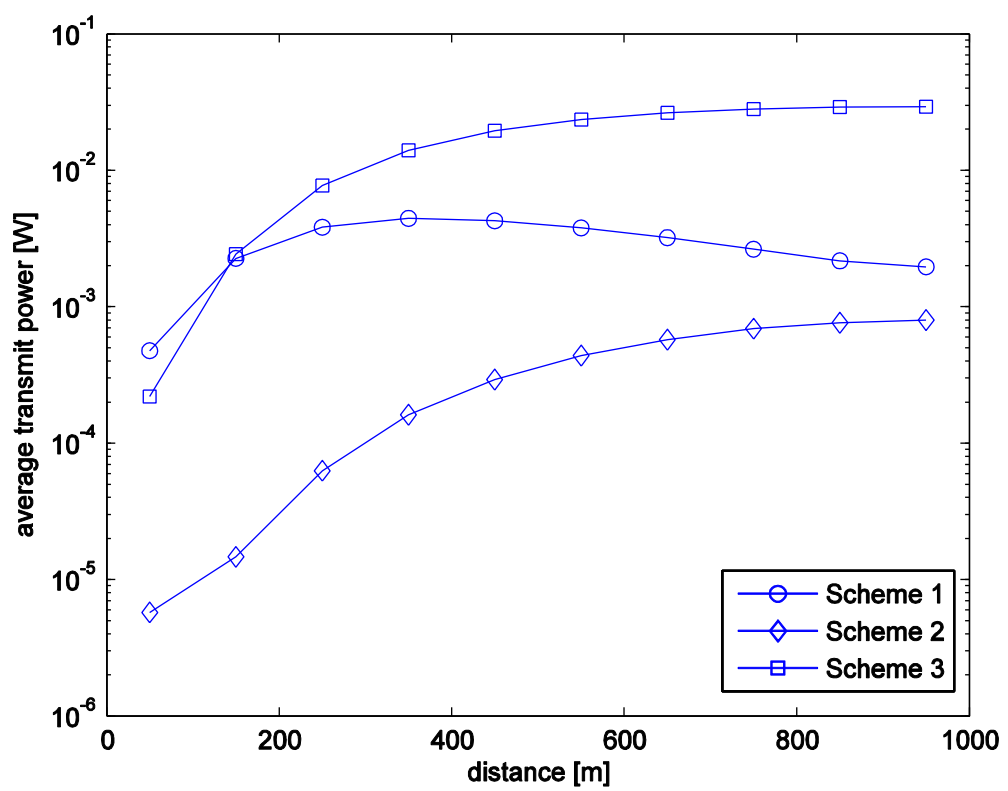

Fig. 3. Average transmit power as a function of the user distance 


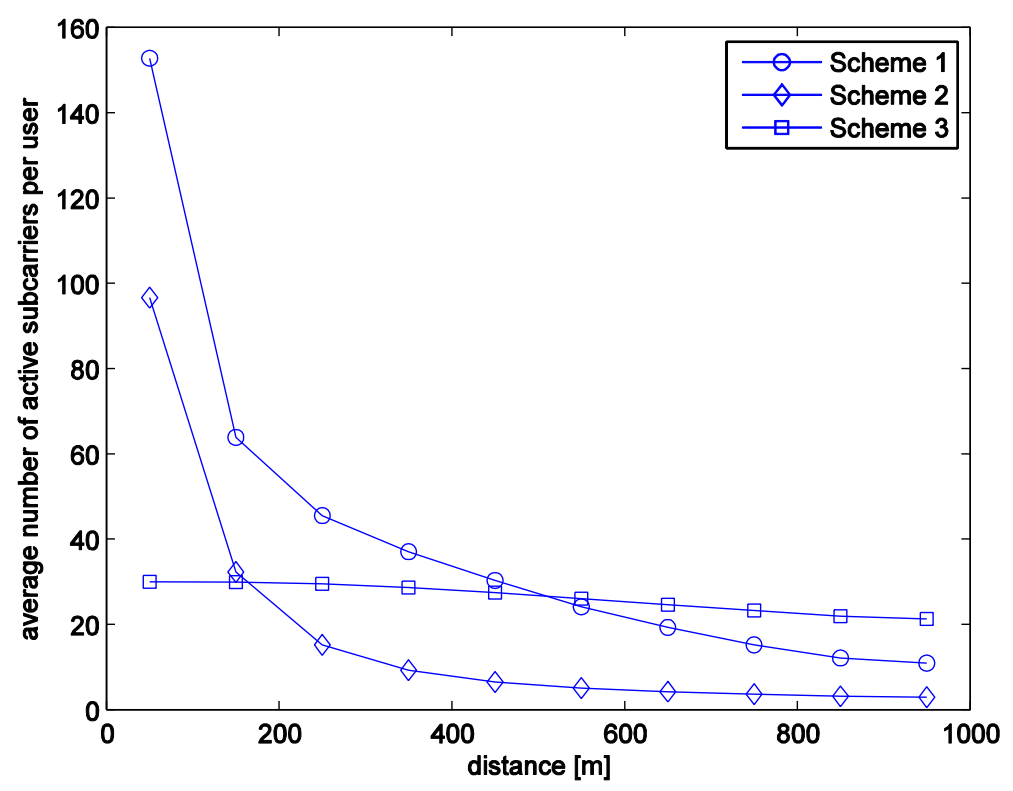

Fig. 4. Average number of active subcarriers as a function of the user distance

Another interesting result comes from the shape of the transmit power curve for scheme 1 . Unlike schemes 2 and 3 , the transmit power of scheme 1 decreases for distances greater than $350 \mathrm{~m}$, which means that the number of subcarriers allocated to users at those distances by scheme 1 decreases significantly.

The number of active subcarriers per user, depicted in Fig. 4, gives us information about the PRB allocation method of each scheme. As can be seen, scheme 1 and scheme 2 tend to allocate a greater number of subcarriers to users near the base having a drastic drop down in this number for the farther users. However, users in scheme 1 have always more active subcarriers than users in scheme 2 which indicates that the introduction of the threshold $\lambda$ for allocation in scheme 1 helps edge users to improve its performance. In contrast, scheme 2 doesn't have any notion of justice as it tries to maximize the overall energy efficiency sum. Besides, scheme 2's centralized allocation guarantee that all users receive the same number of PRBs and the slight decrease for farther users only indicates that their channel conditions are too bad to transmit in some of the allocated PRBs.

Finally, Fig. 5 presents the average no-transmission probability, which has not been reported for any of the compared schemes. It is noted that schemes 1 and 2 have no-transmission probabilities that are above $90 \%$ while scheme 3 maintains it below $20 \%$. These results are directly related to the way in which PRBs are allocated and the way in which the interference is managed. In the first two schemes most PRBs are allocated to users with the best channel conditions which turn out to be the users near the base, while the farther users receive less PRBs as discussed above. Furthermore, these two schemes try to mitigate interference only through power control. On the 
Performance Evaluation of Energy-Efficient Game-Theoretic Resource Allocation Schemes ...

other hand, scheme 3 performs an equal number allocation in a centralized manner; in this way, it can control the interference by means of both PRB allocation and power control. Analyzing these results we note that each scheme implies a different trade-off between energy efficiency, fairness and no-transmission probability. However, the need of a novel scheme that reduces the no-transmission probability while maintaining the energy efficiency and throughput is evidenced.

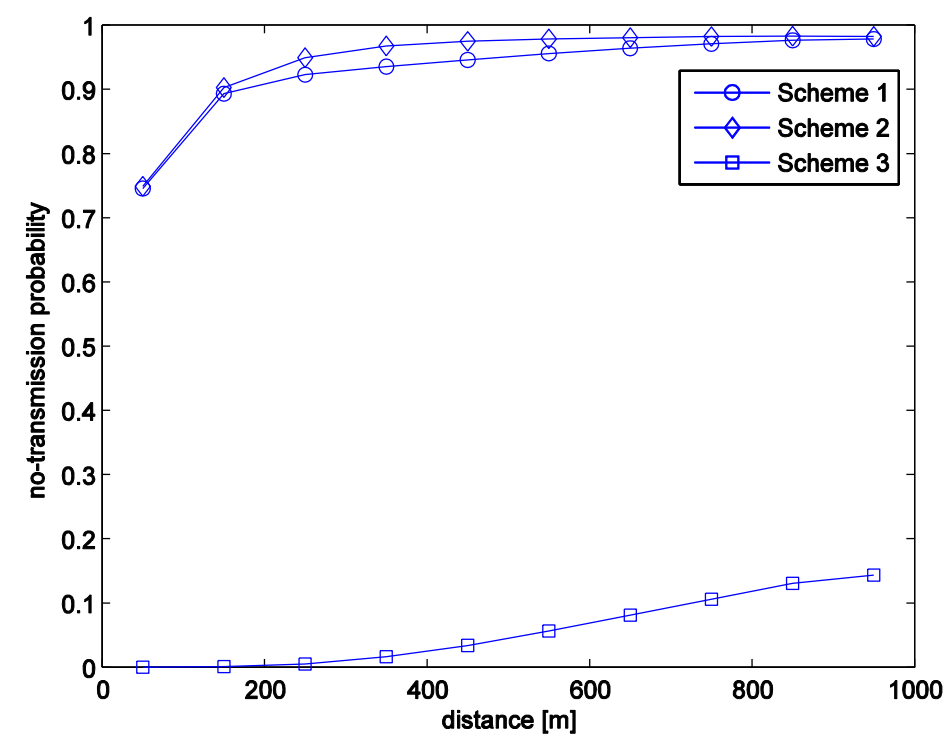

Fig. 5. No-transmission probability as a function of the user distance

\section{Conclusions}

A comparison considering a common system model and a realistic simulation environment between three different energy efficient game-theoretic resource allocation schemes is presented in this paper. The results show the tradeoffs in the different schemes, evidencing the need of a novel scheme which improves the fairness of the system by reducing the no-transmission probability while maintaining the energy efficiency per user.

\section{References}

1. Wong, C.Y., Cheng, R.S., Letaief, K.B., Murch, R.D.: Multiuser subcarrier allocation for OFDM transmission using adaptive modulation. In: IEEE Vehicular Technology Conference, pp. 479-483. IEEE Press, Texas (1999) 
2. Tham, M. L., Chow, C.O., Utsu, K., Ishii, H.: BER-driven resource allocation in OFDMA systems. In: IEEE 24th International Symposium on Personal Indoor and Mobile Radio Communications (PIMRC), pp.1513-1517, IEEE Press, San Francisco (2013)

3. Hanly, S.V., Andrew, L.L.H., Thanabalasingham, T.: Dynamic Allocation of Subcarriers and Transmit Powers in an OFDMA Cellular Network. IEEE Transactions on Information Theory, vol. 55, no. 12, 5445-5462 (2009)

4. Yiwei, Y., Dutkiewicz, E.; Xiaojing H., Mueck, M.: Downlink Resource Allocation for Next Generation Wireless Networks with Inter-Cell Interference. IEEE Transactions on Wireless Communications, vol. 12, no. 4, 1783-1793 (2013)

5. Wei, Y. Wonjong, R., Boyd, S., Cioffi, J.M.: Iterative water-filling for Gaussian vector multiple-access channels. IEEE Transactions on Information Theory, vol. 50, no. 1, 145$152(2004)$

6. Ya-Feng L., Yu-Hong D.: On the Complexity of Joint Subcarrier and Power Allocation for Multi-User OFDMA Systems. IEEE Transactions on Signal Processing, vol. 62, no. 3, 583-596 (2014)

7. Fudenberg, D., Tirole, J.: Game Theory. MIT Press, Cambridge, MA (1991)

8. Hui, L., Wei, Z., Haijun, Z., Zhicai, Z., Xiangming, W.: An iterative two-step algorithm for energy efficient resource allocation in multi-cell OFDMA networks. In: Wireless Communications and Networking Conference (WCNC), pp. 608-613, IEEE Press, Shangai (2013)

9. Alavi, S.M., Chi, Z., Wan, W. G.: Efficient and Fair Resource Allocation Scheme for OFDMA Networks Based on Auction Game. In IEEE Vehicular Technology Conference, pp.1-5, IEEE Press Quebec (2012)

10. Buzzi, S., Colavolpe, G., Saturnino, D., Zappone, A.: Potential Games for EnergyEfficient Power Control and Subcarrier Allocation in Uplink Multicell OFDMA Systems. IEEE Journal of Selected Topics in Signal Processing, vol. 6, no. 2, 89-103 (2012)

11. Dan W., Liang Z., Yueming C., Rodrigues, J.: Energy-efficient resource allocation for uplink OFDMA systems using correlated equilibrium. In: Global Communications Conference (GLOBECOM), pp.4589-4593, Anaheim (2012)

12. Chieh Y. H., Ching-Yao H.: Non-Cooperative Multi-Cell Resource Allocation and Modulation Adaptation for Maximizing Energy Efficiency in Uplink OFDMA Cellular Networks. Wireless Communications Letters, vol.1, no.5, 420-423 (2012)

13. Bacci, G., Bulzomato, A., Luise, M.: Uplink power control and subcarrier assignment for an OFDMA multicellular network based on game theory. In: Int. Conf. Perf. Eval. Methodol. Tools (ValueTools), Paris (2011)

14. Jing, Q., Zheng, S.: Distributed Resource Allocation Based on Game Theory in Multi-cell OFDMA Systems. International Journal of Wireless Information Networks. Vol. 16 . No. $1-2,44-50$ (2009)

15. 3GPP TR 25.814.: Physical layer aspects for evolved UTRA (release 7), 3GPP (2006)

16. Goodman, D., Mandayam, N.: Power control for wireless data. IEEE Personal Communications, vol. 7, no. 2, 48-54 (2000)

17. 3GPP TR 25.892.: Feasibility study for OFDM for UTRAN enhancement (release 6), 3GPP (2004)

18. Lin, Y.B., Mak, V. W.: Eliminating the boundary effect of a large-scale personal communication service network simulation. ACM Transactions on Modeling and Computer Simulation, vol. 4, no. 2, (1994)

19. ITU-R. M.1225.: Guidelines for Evaluation of Radio Transmission Technologies for IMT2000: ITU-R (1997) 